\title{
SHOCKLIKE SOLUTIONS OF THE KORTEWEG-DE VRIES
}

EQUATION

M.Q. Tran, K. Appert, Ch. Hollenstein,

R.W. Means and J. Vaclavik

Centre de Recherches en Physique des P1asmas

ECOLE POLYTECHNIQUE FEDERALE DE LAUSANNE 


\title{
SHOCKLIKE SOLUTIONS OF THE KORTEWEG-DE VRIES
}

EQUATION

\author{
M.Q. Tran, K. Appert, Ch. Hollenstein, \\ R.W. Means and J. Vaclavik
}

ABSTRACT

Plasma response to a piston driver has been modelled by the Korteweg-de Vries equation. The numerical shocklike solutions are evolving solitons, similar to the asymptotic solutions of Gurevich and Pitaevski (1974). Quantitative experimental agreement is found with low-amplitude shock like structures in our DP device.

Collisionless laminar ion acoustic shock waves and solitons have been described by many plasma models. The simplest model which can include the dynamics is contained in the weakly nonlinear Korteweg-de Vries equation (Washimi and Taniuti, 1966). The ions are cold and described by the dissipationless fluid equations. The electrons are hot and in thermal equilibrium with the wave potential. The Korteweg-de Vries equations describes small amplitude nonlinear waves moving with velocity near the ion acoustic speed. Convective steepening and dispersion are included in the equation. This model does not have a stationary shock wave. However, the 
asymptotic solutions of Gurevich and Pitaevski indicate that shocklike structures are possible with a piston driver.

If the ions have a finite temperature then some of them can be reflected by the wave front. Moiseev and Sagdeev (1963) constructed such a model which has a steady state shock wave solution. Their model, however, says nothing about the time evolution of the shock waves. White et al. (1974) considered the nonlinear ion fluid equations with isothermal electrons and numerically solved the piston problem by modelling the potential difference between two chambers of a double plasma device. Their results indicated that a quasi-steady shock structure was formed. That is, behind the shock front, a small number of oscillations existed and the number and character of these oscillations changed only very slowly with time. Their numerical solutions, however, were not followed long enough in time to definitely state that a quasi-steady state structure had been formed, and to compare them with experimental structures (Taylor et a1.,1970). The latter cannot be compared with the asymptotic solutions of Gurevich and Pitaevski (1974) neither since the observation time is too short. The question that remains is whether the observed laminar small amplitude shocklike structures generated in the DP device are shock waves as described by Moiseev and Sagdeev (1963) or are non-linear waves whose structure can be explained by convection and dispersion. In our work a numerical integration of the K.dV equation allows us to follow the evolution of a ramp perturbation within the experimental observation time scale. The obtained result is an evolving soliton train which looks qualitatively very much like the observed shocklike structure. 
The Korteweg-de Vries equation has been solved using two different aproaches. In the first one we define the stretched coordinates as $\eta=x$ and $\xi=x-t$. The piston problem is then a boundary value problem where the perturbation density is fixed at $\eta=0$. Numerical solutions are computed using the Zabusky (1968) left moving scheme with the following boundaries:

$$
\begin{aligned}
& n(\eta, \xi=L)=0=\left.\frac{\partial n}{\partial \xi}\right|_{\xi=L} \\
& n(n, \xi=0)=n(\eta=0, \xi=0) \\
& n(\eta=0,-\xi)=n(x=0, t) .
\end{aligned}
$$

In the second approach, we include in the right hand side of the Kortewegde Vries equation a source term which describes the injection of particles from the driver plasma into the target plasma. The stretched coordinates are defined as $\eta=t$ and $\xi=x-t$ and the source of strength $s(\eta)$ is located at $\xi=\xi_{0}+\eta$. With these definitions, the Korteweg-de Vries equation is written as:

$$
\frac{\partial n}{\partial \eta}+n \frac{\partial n}{\partial \xi}+\frac{1}{q} \frac{\partial^{3} n}{\partial \xi^{3}}=S(\eta) \delta\left(\xi-\xi_{0}-\eta\right)
$$

with the initial value $n(\eta=0, \xi)=0$. Equation (1) is then Fourier analysed in $\}$ in a box of the length $L$ and the evolution of each mode is then computed as function of $\eta$ until the solution reaches the boundary.

In both cases, the boundary value $n(\eta=0, \xi)$ and the source term $s(\eta)$ respectively vary in real time $t$ as a ramp with a rise time of the order of 25-50 $\omega_{\mathrm{pi}}^{-1}$. Both methods used give the same evolution of the perturbation in the target chamber. A typical run is shown in Fig. 1. The 
perturbation steepens and oscillations develope at the leading edge. As $\eta$ (i.e. $x$ or $t$ ) is increased more oscillations emerge. Eventually the separation of the first oscillation is achieved and its properties, remain nearly unchanged for large $\tau$. Finally the number of oscillations gradually increases forming an oscillatory structure behind the leading edge. At this stage the perturbation does not depend on the rise time of the boundary value or source term, but only on its amplitude. The computed structure is nearly identical to the experimental shocklike structure reported by Taylor (1970) and Taylor et al. (1970).

In Fig. 2 we have reported the dependency of the Mach number and half width $\mathrm{D}$ (measured at $.42 \mathrm{x}$ maximum amplitude) for the first oscillation. There is a close fit between the numerical results and the well known soliton's characteristics (Washimi and Taniuti, 1966). The same dependency is noted for the next oscillation for large enough $\tau$. Therefore each oscillation can be identified as a soliton and the whole structure is an ensemble of evolving solitons.

A characteristic feature of the observed structure is that the amplitude of the first soliton is twice the height of the driver (Gurevich and Pitaevski, 1974). Using other models of stationary shocks with dissipation one finds an oscillatory structure which damps to a level which depends on the damping rate (Taylor, 1970, Means, 1972, Whitham, 1974). This relation has been compared with experimental data from our DP device. The machine is operated at a plasma density of $10^{9} \mathrm{~cm}^{-3}$, an electron temperature of 1 $\mathrm{eV}$ and an ion temperature of .1 eV (Hirt and Tran, 1974). The shocklike 
perturbation is produced by applying a ramp to the driver chamber. The signal from a plane Langmuir probe $(\emptyset 5 \mathrm{~mm})$ is feed into a boxcar integrator (PAR 162) and then recorded on $X Y$ recorder for different probe position. For low amplitude excitation ( $\delta_{n} \sim 10-20 \%$ ) the shocklike structure reflects only a small amount of ions so that the ions can be considered to be cold. For these structures the amplitude of the first soliton is twice the amplitude of the level behind the wave train as shown in Fig. 3. However, when the amplitude of the perturbation is increased ( $\delta \mathrm{n} \sim 30 \%$ and more), the number of reflected ions increases and turbulence develops at the shock foot. The Korteweg-de Vries equation is no longer applicable and one would expect another dependency between these two quantities. Data obtained previously by Means et al. (1973) confirm this last statement (Fig. 3).

In conclusion, it is possible to state that:

1) For the piston problem, the Korteweg-de Vries equation has solutions consisting of a train of solitons, the maximum amplitude of which is twice the height of the piston. This train of solitons looks like a shock. However, it must not be confused with Sagdeev's shock since it is not stationary and does not present any change in entropy. (In Sagdeev's model, entropy increase is provided by the ion reflection).

2) Experiments conducted in DP devices show that under certain conditions (small amplitude perturbation so that there are few reflected ions) the shocklike structure can be identified with a train of evolving solitons. 
ACKNOWLEDGMENT

The authors are grateful for the many helpful discussions with Doctor J.M. Greene.

This work was supported by the Swiss National Science Foundation. 
REFERENCES

Gurevich A.V. and Pitaevski L.P. (1974) Sov.Phys.JETP 38, 291

Hirt P.J. and Tran M.Q. (1974) HPA 47, 475

Means R.W. (1972) UCLA Report PPG 115

Means R.W., Coroniti F.V., Wong A.Y. and White R.B. (1973) Phys.Fluids 16, 2304

Moiseev G.S. and Sagdeev R.Z. (1963) J.Nuc1.Energy Part e5, 43

Taylor R.J. (1970) UCLA Report PPG 70

Taylor R.J., Baker D.R. and Ikezi H. (1970), Phys.Rev.Lett. 24, 206

Washimi H. and Taniuti T. (1966) Phys.Rev.Lett. 17, 966

White R.B., Fried B.D. and Coroniti F.V. (1974) Phys.Fluids 17, 211

Whitham G.B. (1974) Linear and Non Linear Waves (John Wiley and Sons) p. 484 
Figure 1 : Shocklike structures generated by Korteweg-de Vries equation. The source is at $x=0$.

Figure 2 : Comparison of numerical results with theoretical soliton characteristics. Solid line is $M-1=0.3 \delta \mathrm{n} / \mathrm{n}_{0}$, where $M$ is the Mach number. Dotted line is $D=\sqrt{6 n_{0} / \delta n}$, where $D$ is the soliton half-width. $\Delta$ and 0 , results from boundary value solutions.

$\Delta$ and $\mathbf{Q}$, results from source term solutions.

Figure 3 : Amplitude of the first soliton (at large time) versus ramp amplitude. Solid line is $\delta_{n} / n_{0}=2 \cdot($ ramp amplitude $)++$, experimental results for small amplitude excitations. 0, results from boundary value solutions. 0 , results from source term solutions. $\Delta$, previous results from turbulent shocks ${ }^{6}$. 


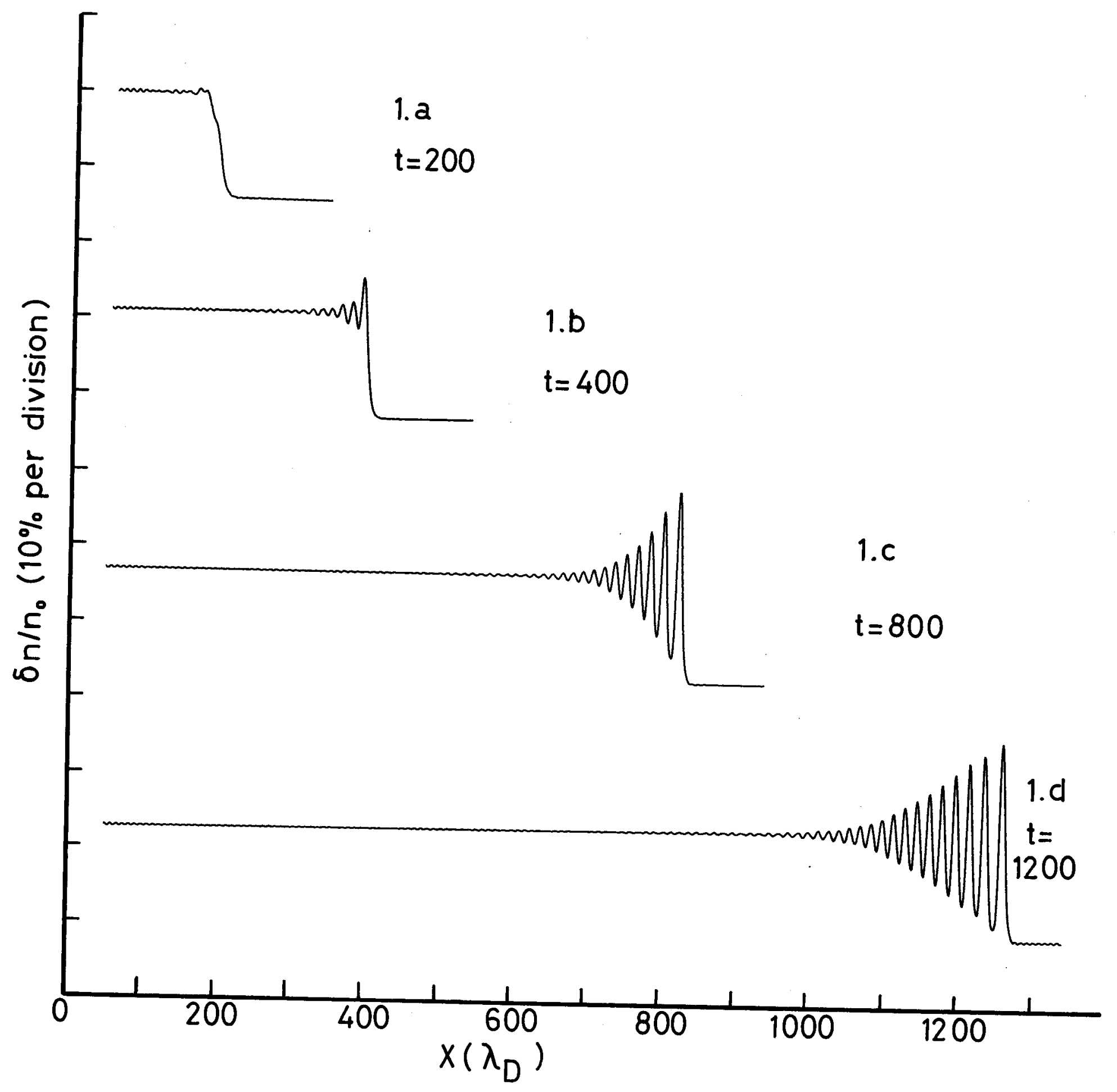

Fig $\cdot \mathbf{l}$ 


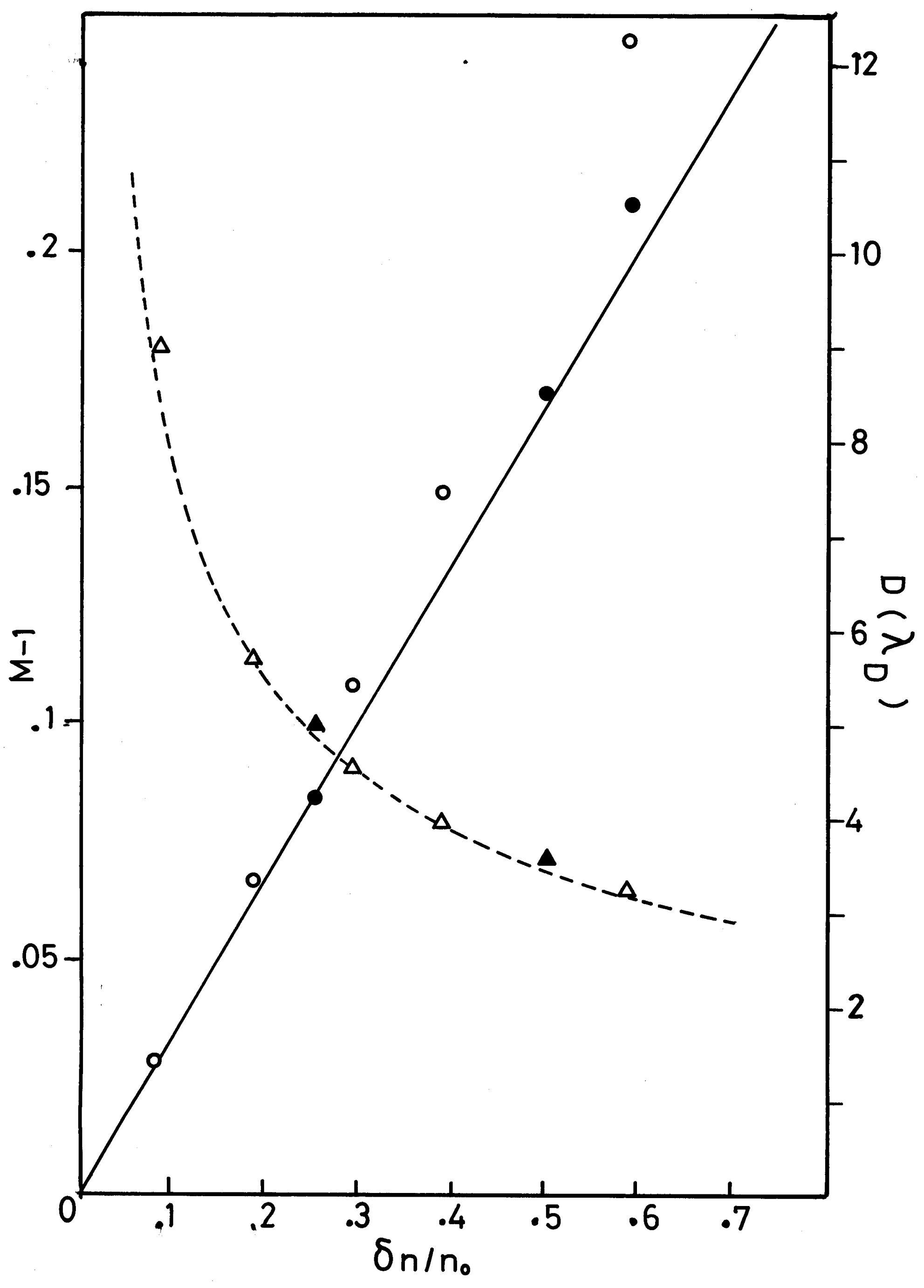




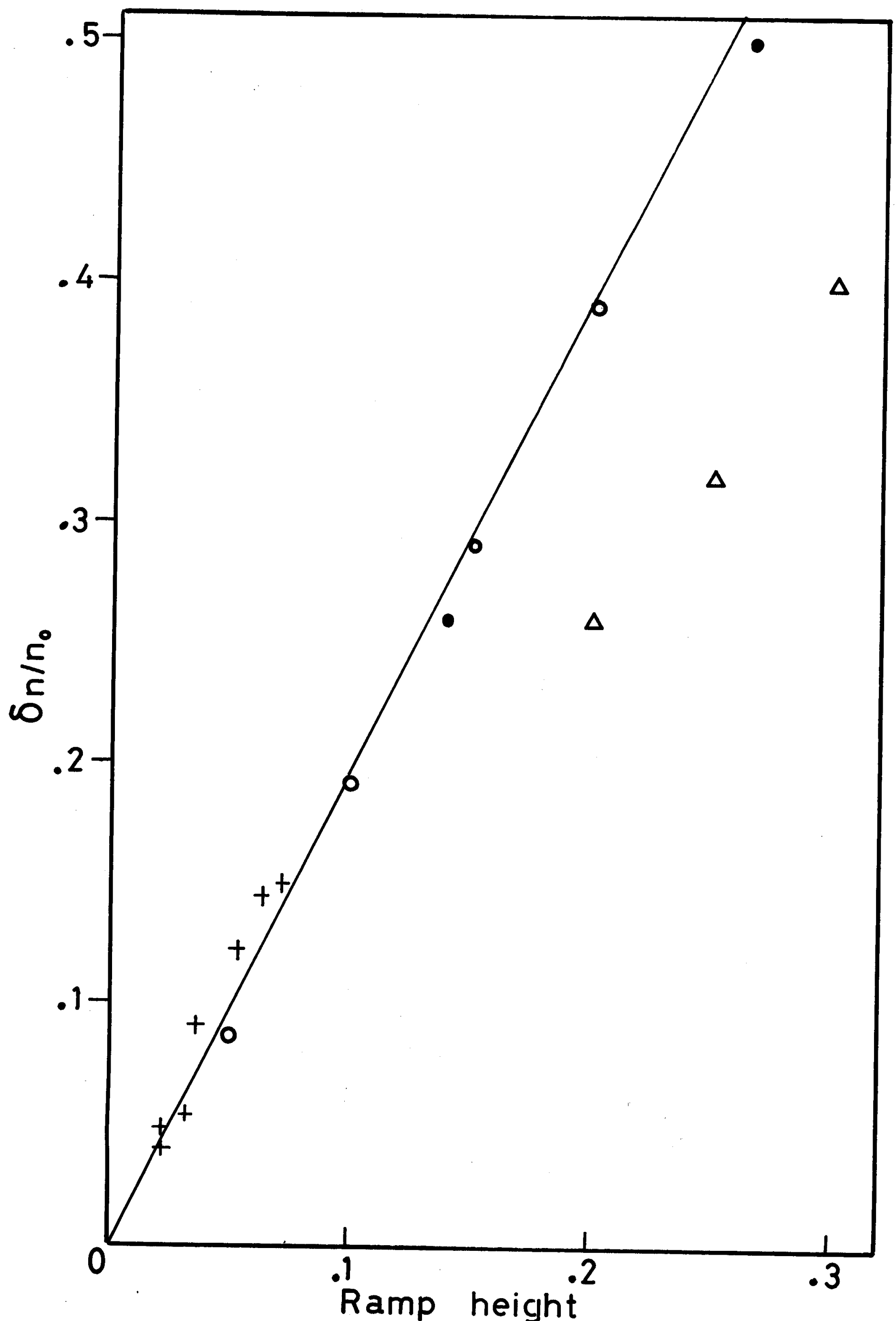

